International Journal of Modern Physics E

(C) World Scientific Publishing Company

\title{
NON-STRANGE MESON PRODUCTION WITH HADRONIC PROBES
}

\author{
C. HANHART \\ Institut für Kernphysik and Jülich Center for Hadron Physics, \\ Forschungzentrum Jülich, D-52425 Jülich, Germany \\ c.hanhart@fz-juelich.de
}

Received 11 September 2008

Revised 24 September 2008

Recent progress in the field of meson production in hadron collisions is presented. Special emphasis is put new developments in the application of effective field theory methods to pion production, but also theoretical concepts for two-pion, $\eta$, and vector-meson production will be presented.

\section{Introduction}

As a consequence of the large coupling strength of Quantum-Chromo Dynamics (QCD) at low and intermediate energies, standard perturbative methods fail in fact, the interaction gets so strong at increasing distances that it confines all color-charged particles (quarks and gluons) into areas of the order of $10^{-15} \mathrm{~m}$ only those particles that are neutral with respect to the color force (these are called hadrons) are allowed to travel longer distances. Due to this complication, strong QCD is one of the last terrae incognitae of the Standard Model.

To theoretically approach this interesting regime there are currently three options available: One is to use so called QCD inspired quark models. Especially in spectroscopy they are widely used and lead to very useful insights on hadron structure - see Ref. 1. On the other hand, the application of those approaches to hadron dynamics is technically very involved and often only lead to a qualitative agreement with the phenomenology. Theoretically more sound is to solve QCD numerically with large scale simulation in a discretized space. This method is called lattice QCD. A lot of progress was made in recent years. E.g. in Ref. 2 the glueball spectrum is calculated in pure Yang-Mills theory. In addition, first results for hadron-hadron scattering processes at low energies were reported recently 3 .

The third alternative is to work directly with the physical degrees of freedom relevant at the given energy, namely with hadrons. On the first glance it seems as if in this approach the connection to QCD were lost, however, with the advent of effective field theories some 30 years ago 4 this connection became well established. It is nowadays possible to preform calculations with hadronic degrees of freedom with 
high accuracy and contolled uncertainty. The probalbly most impressive amongst those works is the recent determination of the $\pi-\pi$ scattering lengths using chiral perturbation theory $(\mathrm{ChPT})$ - the effective field theory for the standard model at low energies - in combination with dispersion theory 5 .

The reason why it became possible to study QCD by looking at hadronic observables directly is its approximate $S U(2)_{L} \times S U(2)_{R}$ symmetry, spontaneously broken to $S U(2)_{V}$. This mechanism leads to the appearance of the three pions as pseudoGoldstone bosons that are interacting only weakly at low energies. In consequence there is, at low energies, a separation of scales between the pion mass/momenta and the typical hadronic scale set by $\Lambda_{\chi} \simeq 4 \pi f_{\pi} \simeq M_{N} \simeq 1 \mathrm{GeV}$, where $f_{\pi}$ and $M_{N}$ denote the pion decay constant and the nucleon mass, respectively. In addition, the structure of the interactions of pions with each other as well as heavy fields is largely controlled by the above mentioned symmetry group. The resulting effective field theory is the above mentioned ChPT. For a recent review see 6 .

The standard expansion parameters of ChPT are $m_{\pi} / \Lambda_{\chi}$ and $q / \Lambda_{\chi}$, where $m_{\pi}$ and $q$ denote the pion mass and a typical momentum of the reaction, respectively. ChPT is a non-renormalizable field theory, however, by construction it can be renormalized with a finite number of counter terms - the so called low energy constants (LECs) - at each order in the expasion. As a consequence with increasing orders new parameters appear in the theory. Ideally those are to be fixed from experiment directly. In case of a lack of experimental information or in order to understand the phenomenological content of LECs, their values may be determined from matching to models that contain heavier degrees of freedom explicitly. This program, called resonance saturation, was carried through successfully for $\pi \pi$ scattering ${ }^{7}, \pi N$ scattering $[8$, and $N N$ scattering 9 . In any case the predictive power of the theory emerges from the fact the the same LECs contribute to very different reactions. Examples of this will be given below.

\section{2. $N N \rightarrow N N \pi$}

A first step to calculate elastic and inelastic pion reactions on the few nucleon system was taken by Weinberg already in 1992 10. He suggested that all that needs to be done is to convolute transition operators, calculated perturbatively in standard chiral perturbation theory (ChPT), with proper nuclear wave functions to account for the non-perturbative character of the few-nucleon systems. This procedure combines the distorted wave born approximation, used routinely in phenomenological calculations, with a systematic power counting for the production operators. Within ChPT this idea was already applied to a large number of reactions like $\pi d \rightarrow \pi d^{11}$, $\gamma d \rightarrow \pi^{0} d^{12[13}, \pi^{3} \mathrm{He} \rightarrow \pi^{3} \mathrm{He} \stackrel{14}{ }, \pi^{-} d \rightarrow \gamma n n$ 15, and $\gamma d \rightarrow \pi^{+} n n$ 16117, where only the most recent references are given.

The central concept to be used in the construction of the transition operators is that of reducibility, for it allows one to disentangle effects of the wave functions and those from the transition operators. As long as the operators are energy in- 
dependent, the scheme can be applied straight forwardly 18 , however, for energy dependent interactions more care is necessary, for typically induced non-reducible pieces appear. For the reaction $p p \rightarrow d \pi^{+}$this is demonstrated in detail in Ref. 19, where a subtle interplay between these and other one loop integrals was found.

Using standard ChPT especially means to treat the nucleon as a heavy field. Corrections due to the finite nucleon mass, $M_{N}$, appear as contact interactions on the lagrangian level that are necessarily analytic in $M_{N}$. However, some pion-fewnucleon diagrams employ few-body singularities that lead to contributions nonanalytic in $m_{\pi} / M_{N}$, with $m_{\pi}$ for the pion mass. In Ref. 20 it is explained how to deal with those.

A problem was observed when the original scheme by Weinberg was applied to the reactions $N N \rightarrow N N \pi$ 21|22|23: Potentially higher order corrections turned out to be large and lead to even larger disagreement between theory and experiment. For the reaction $p p \rightarrow p p \pi^{0}$ one loop diagrams that in the Weinberg counting appear only at NNLO where evaluated 24|25 and they turned out to give even larger corrections putting into question the convergence of the whole series. However, already quite early the authors of Refs. $\frac{26 \mid 27}{2}$ stressed that an additional new scale enters, when looking at reactions of the type $N N \rightarrow N N \pi$, that needs to be accounted for in the power counting. Since the two nucleons in the initial state need to have sufficiently high kinetic energy to put the pion in the final state onshell, the initial momentum needs to be larger than

$$
p_{\mathrm{thr}}=\sqrt{M_{N} m_{\pi}} \longrightarrow p_{\mathrm{thr}} / \Lambda_{\chi} \simeq 0.4 .
$$

The proper way to include this scale was presented in Ref. $\frac{28}{28}$ and implemented in Ref. 29 - for a recent review see Ref. 30 . As a result, pion $p$-waves are given by tree level diagrams up to NNLO in the modified power counting and the corresponding calculations showed satisfying agreement with the data 28 . However, for pion $s^{-}$ waves loops appear already at NLO. Those were studied in detail in Ref. 19. The loops turned out to undergo sizable cancellations. The net effect of going to NLO was that the most important operator (upper diagram of Fig. 2, left panel), first investigated in Ref. 31 got enhanced by a factor of $4 / 3$ which was sufficient to overcome the apparent discrepancy with the data. Since the Delta-nucleon mass difference, $\Delta$, is numerically of the order of $p_{t h r}$, also the Delta-isobar should be taken into account explicitly as a dynamical degree of freedom 26 _ in line with phenomenological findings $32 \mid 33$.

Once the reaction $N N \rightarrow d \pi$ is understood within effective field theory one is in the position to also calculate the so-called dispersive and absorptive corrections to the $\pi d$ scattering length. With these results, presented in Ref. 34/35, a high accuracy determination of the $\pi N$ scattering lengths using information on $a_{\pi d}$ becomes possible - for a review of the latter subject we refer to Ref. 37 .

In Ref. 28 it was stressed that there is a close connection between the leading $(\bar{N} N)^{2} \pi$ counter term and an important term in the three-nucleon force. The latter

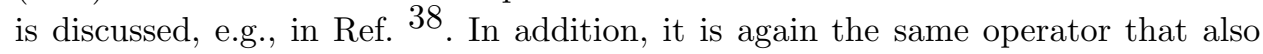




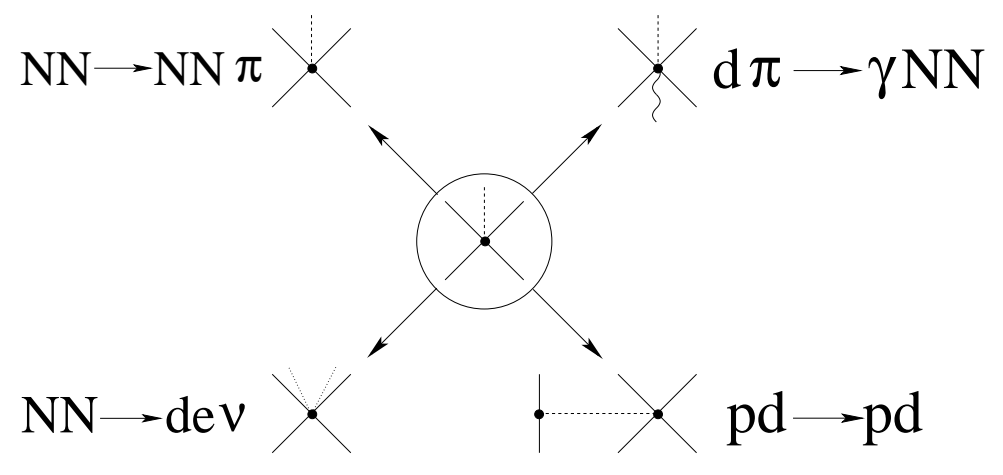

Fig. 1. Illustration of the various reactions, where the leading $4 N \pi$-contact term contributes.

appears in $\gamma d \rightarrow \pi^{+} N N$ and $\pi d \rightarrow \gamma N N \underline{39}$ as well as in weak reactions like tritium beta decay 40|41. The appearance of the same operator in various reactions is illustrated in Fig. 11 Note that the same operator appears in all these reactions in very different kinematics ranging from very low energies for both incoming and outgoing $N N$ pairs in $p d$ scattering and the weak interactions up to relatively high initial energies for the $N N$ induced pion production. In Ref. 42 an aparent discrepancy between the strength of the counter term needed in $p p \rightarrow p n \pi^{+}$and in tritium beta decay was reported. This difference might indicate a non-applicability of ChPT to the reactions $N N \rightarrow N N \pi$. On the other hand, in the partial wave analysis of the $p p \rightarrow p n \pi^{+} 43$ it was assumed that the isospion $1 N N$ final state contributes in the $S$-wave only. However, it was shown in Refs. $\frac{44 \mid 45}{4}$ that is assumption is not justified. Further experimental and theoretical studies are clearly called for to resolve this issue. Here the planned double-polarization measurements at COSY are very important 46 .

As mentioned above the mechanism of spontaneous symmetry breaking constraints the interactions of pions in a very non-trivial way. One of the most striking examples is that the contributions to the proton-neutron mass difference get linked to the leading, isospin violating $\pi N$ scattering $47 / 48$. Since it is possible to manipulate the total isospin of a hadronic reaction using light nuclei either in the final or the initial state, pion production in $N N$ and $d d$ collisions proved as ideal to study these isospin violations. The measurements of the forward-backward assymmetry in $p n \rightarrow d \pi^{0} 49$ and of the total cross section for $d d \rightarrow \alpha \pi^{0}[50$ show clearly the presence of isospin violation also in hadron dynamics beyond effects resulting from pion mass differences. Theoretically first steps for a consistent analysis of the two reactions were taken in Ref. 51 and Refs. 52 , respectively. It is important to note that the operator structure of the leading operators in the reactions mentioned above, which is fixed by the assumed symmetry properties of the QCD+QED, also appears in isospin violating decays of $D_{s}(2317)$ mesons $[53$. This again is a good ex- 


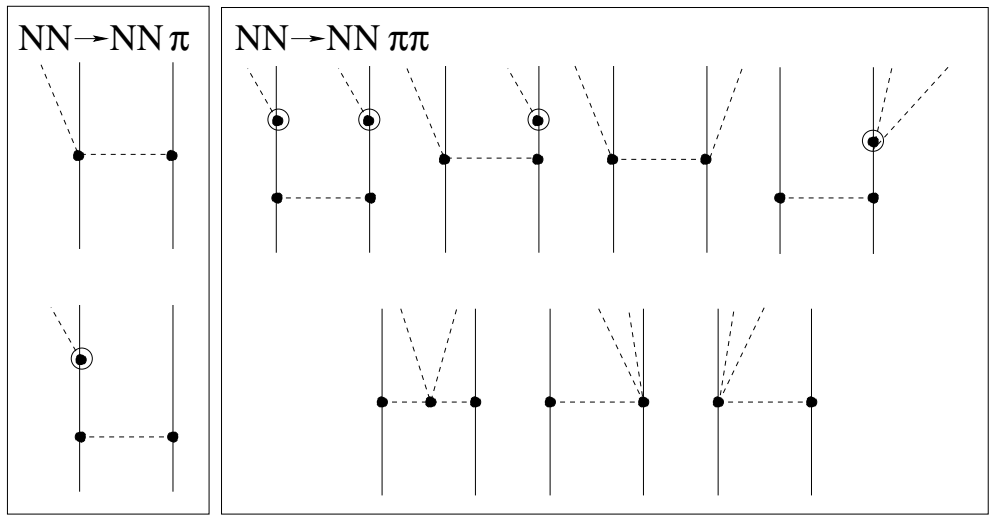

Fig. 2. Leading nucleonic diagrams that contribute to $N N \rightarrow N N \pi$ (left panel) and $N N \rightarrow N N \pi \pi$ (right panel) near threshold. Solid (dashed) lines denote nucleons (pions), solid dots (encircled dots) denote vertices from the leading (next-to-leading) Lagrangian.

ample how effective field theories by connecting different reactions allow for deeper insights into the underlying theory. In order to establish this connection better data for both systems is needed. In case of $d d \rightarrow \alpha \pi^{0}$ this will be provided by WASA at COSY $\left[54\right.$, in case of the $D_{s}$ decay by PANDA at FAIR.

\section{Two-pion production}

In recent years the reactions $N N \rightarrow N N \pi \pi$ was studied both phenomenologically 55 as well as experimentally $\underline{56 / 57 / 58}$. However, so far no effective field theory calculation exists for this class of reactions.

In case of two-pion production the threshold momentum is even larger and accoringly the expansion parameter relevant for reactions of the type $N N \rightarrow N N \pi \pi$ is $p_{\mathrm{thr}} / \Lambda_{\chi}=0.54$, which makes the applicability of ChPT here questionable. However, it is still instructive to investigate the structure of diagrams that contribute at the lowest orders. In the right panel of Fig. 2 the nucleonic diagrams are shown that contribute to leading order to two-pion production in nucleon-nucleon collisions at threshold. Note that at leading order for some of these there are counter parts that contain the Delta-isobar.

For comparision in the left panel we show those that contribute to leading order to $N N \rightarrow N N \pi$. Obviously, the number of diagrams that contributes increased significantly. It remains to be seen how well this set of amplitudes describes the production amplitude near threshold. In this context it is important to note that the phenomenological amplitudes of Ref. 55 are not able to explain the empirical data 57/58. In this work only some of the diagrams shown above are included.

Again, already at next-to-leading order loops start to contribute. In the two pion production those consist of two sucessive one pion emissions. These loops are 
known to be finit@ and do not introduce any new parameters. At $\mathrm{N}^{2} \mathrm{LO}$, additional loops contribute. Those are divergent and, consequently, at this order the first counter-terms enter the calculation. Note, the concept of resonance saturation allows one to identify these counter terms with some of the baryon resonance contributions that were included in the calculations of Ref. 55 . In calculations for single pion production in $N N$ collisions the Delta isobar needs to be kept as a dynamical degree of freedom. Tree level diagrams including the Delta therefore appear already at rather low orders. It remains to be seen, if a similar promotion of resonace diagrams is necessary for additional resonances in case of the two pion production. A good candidate for this might be the Roper resonance that is believed to have a significant two pion contribution 59 which results in a sizable empirical coupling to the two-pion channels $60|55| 61$.

Nowadays a large number of differential and even polarized data are available for the two pion reactions in nucleon-nucleon collisions 58 . It will be very important to investigate the interplay of the diagrams controlled by chiral symmetry (see, e.g., Fig. (2) and the resonance contributions as the excess energy increases. Especially to analyse the polarization data a proper inclusion of final and initial state interactions is essential, for analysing powers are given by the imaginary parts of interference terms and the $N N$-distorsions are the essential source of non-vanishing phases. These issues will be investigated in the coming year.

\section{Production of heavier mesons}

Was it already questionable, if the chiral expansion can still be applied for the two pion production processes, it is out of question that there is no way to use it to study the production of heavier mesons. Already for $\eta$ production, we get $p_{\mathrm{thr}} / \Lambda_{\chi} \simeq$ $\sqrt{m_{\eta} / M_{N}}=0.8$. Does this mean that there is no model independent approach possible in this case? Fortunately the answer to this question is no. It is well known that for large momentum transfer reactions the final state interactions become universal 62 . Thus, as soon as one studies a reaction with sufficiently strong final state interactions, it is possible to disentangle those from the production operator in a model-intependent fashion. The signal of the final state interaction is a distorsion of the invariant mass distributions of outgoing particles. It should be stressed that it is non-trivial to make the connection of the signal of final-state interactions to the scattering parameters quantitative 63 (for elastic final state interactions a method derived in Ref. 64 ) and that in the presence of such strong final state interactions a determination of the strength of the total production amplitude seems not to be possible model independently 65 .

A very nice example for the given discussion is the observation of the strong $\eta^{3} \mathrm{He}$ interaction in the reactions $\gamma^{3} \mathrm{He} \rightarrow \eta^{3} \mathrm{He}\left[66\right.$ and $p d \rightarrow \eta^{3} \mathrm{He} \underline{67|68| 69}$ - see also talk by $\mathrm{A}$. Khoukaz at this conference. In both reactions a very pronounced

a The same class of diagrams was studied in different kinematics in Refs. 34/35. 
rise in the amplitude is seen very close to the production threshold pointing at a very near-by singularity in the $\eta^{3} \mathrm{He}$ scattering amplitude 70 . In addition, the angular assymmetry shows a very unusal energy dependence 11 . In order to decide whether the $\eta-$ He interaction is sufficiently strong to form a bound state, additional sub-threshold data is necessary. Such data exists $[66$, however, not yet of sufficient accuracy to decide on the subject 72 .

In some cases it is also possible to deduce information on the production operator itself directly from observables even in the case of the production of heavy mesons. A nice example is vector-meson production in nucleon-nucleon collisions. There was the hope that significant deviations from predictions based on the OZIrule 73 in case of strangeness production might allow one to draw conclusions on the strangeness content of the nucleon $74 / 75$. The OZI ratio was also studied in nucleon-nucleon induced reactions via a comparison of $\phi$ and $\omega$ production cross sections. Clearly, although the absolute value of the matrix elements can at present not be controlled theoretically, the ratio of production cross sections contains valuable information that my be extracted from the data using the method explained in Ref. 76 The angular distributions of the $\phi$ production near threshold revealed that the reaction is dominated by a meson exchange current and not by vector meson emission off a single nucleon $77 / 78$ and thus no direct information on the strangeness content of the nucleon can be extracted. On the other hand is a deviation of the expectations of the OZI rule even in this case interesting. In this context it is important to understand the role of resonances in the vector meson production - see, e.g. Refs. 7980 . Although a recent experimental study indicates that the reaction mechanisms might be the same for $\omega$ and $\phi$ production in $N N$ collisions $\frac{81}{}$, further studies, especially with polarized beam, are necessary, before this issue can be resolved. Especially additional information on differential observables might reveal the role played by a possible pentaquark that strongly couples to $\phi p$ ㄷ․

\section{Outlook}

In the years to come significant theoretical progress in the pion production reactions is to be expected. Single pion production will be caluclated up to $\mathrm{N}^{2} \mathrm{LO}$. In addition two- and three-pion production on the two-nucleon system and pion production on few-nucleon systems will be studied. This will pave the way for high accuracy calculations for the isospin violating reactions. For the production of heavier mesons better data will provide deeper insight, e.g., into the interactions of unstable particles.

To summarize, in recent years in the field of non-strange meson production in hadronic collisions significant progress was made on both the theoretical as well as the experimental side. The years to come promise deep insights into the structure and dynamics of strongly interacting particles. 


\section{Acknowledgements}

I would like to thank V. Baru, J. Haidenbauer, A. E. Kudryavtsev, V. Lensky, and U.-G. Meißner for a very educating and productive collaboration.

\section{References}

1. E. S. Swanson, Phys. Rept. 429 (2006) 243 arXiv:hep-ph/0601110.

2. C. J. Morningstar and M. J. Peardon, Phys. Rev. D 60 (1999) 034509 arXiv:hep-lat/9901004.

3. S. R. Beane, T. C. Luu, K. Orginos, A. Parreno, M. J. Savage, A. Torok and A. WalkerLoud, Phys. Rev. D 77 (2008) 014505 arXiv:0706.3026 [hep-lat]].

4. S. Weinberg, Physica Scripta A 96(1979)327; J. Gasser and H. Leutwyler. Ann. Phys. (1984) 158.

5. G. Colangelo, J. Gasser and H. Leutwyler, Nucl. Phys. B 603, 125 (2001) arXiv:hep-ph/0103088.

6. V. Bernard and U.-G. Meißner, Ann. Rev. Nucl. Part. Sci. 57 (2007) 33 arXiv:hep-ph/0611231.

7. G. Ecker, J. Gasser, A. Pich and E. de Rafael, Nucl. Phys. B 321 (1989) 311.

8. V. Bernard, N. Kaiser and U.-G. Meißner, Int. J. Mod. Phys. E 4, 193 (1995) arXiv:hep-ph/9501384.

9. E. Epelbaum, U.-G. Meißner, W. Gloeckle and C. Elster, Phys. Rev. C 65 (2002) 044001 arXiv:nucl-th/0106007.

10. S. Weinberg, Phys. Lett. B 295 (1992) 114.

11. S. R. Beane, V. Bernard, E. Epelbaum, U.-G. Meißner and D. R. Phillips, Nucl. Phys. A 720 (2003) 399. arXiv:hep-ph/0206219.

12. S. R. Beane, V. Bernard, T. S. H. Lee, U.-G. Meißner and U. van Kolck, Nucl. Phys. A 618, 381 (1997) arXiv:hep-ph/9702226.

13. H. Krebs, V. Bernard and U.-G. Meißner, Eur. Phys. J. A 22 (2004) 503 arXiv:nucl-th/0405006.

14. V. Baru, J. Haidenbauer, C. Hanhart and J. A. Niskanen, Eur. Phys. J. A 16, 437 (2003) arXiv:nucl-th/0207040.

15. A. Gardestig and D. R. Phillips, Phys. Rev. C $73 \quad$ (2006) 014002 arXiv:nucl-th/0501049.

16. V. Lensky, V. Baru, J. Haidenbauer, C. Hanhart, A. E. Kudryavtsev and U.G. Meißner, Eur. Phys. J. A 26, 107 (2005) arXiv:nucl-th/0505039.

17. V. Lensky, V. Baru, E. Epelbaum, C. Hanhart, J. Haidenbauer, A. E. Kudryavtsev and U.-G. Meißner, Eur. Phys. J. A 33 (2007) 339 arXiv:0704.0443 [nucl-th]].

18. D. R. Phillips, S. J. Wallace and N. K. Devine, Phys. Rev. C 72 (2005) 0140061. arXiv:nucl-th/0411092.

19. V. Lensky, V. Baru, J. Haidenbauer, C. Hanhart, A. E. Kudryavtsev and U.G. Meißner, Eur. Phys. J. A 27, 37 (2006) arXiv:nucl-th/0511054.

20. V. Baru, C. Hanhart, A. E. Kudryavtsev and U.-G. Meißner, Phys. Lett. B 589, 118 (2004) arXiv:nucl-th/0402027.

21. B.Y. Park et al., Phys. Rev. C 53 (1996) 1519 arXiv:nucl-th/9512023.

22. C. Hanhart, J. Haidenbauer, M. Hoffmann, U.-G. Meißner and J. Speth, Phys. Lett. B 424 (1998) 8 arXiv:nucl-th/9707029.

23. V. Bernard, N. Kaiser and U. G. Meissner, Eur. Phys. J. A 4 (1999) 259 arXiv:nucl-th/9806013.

24. V. Dmitrašinović, K. Kubodera, F. Myhrer and T. Sato, Phys. Lett. B 465 (1999) 43 arXiv:nucl-th/9902048. 
25. S. I. Ando, T. S. Park and D. P. Min, Phys. Lett. B 509 (2001) 253 arXiv:nucl-th/0003004.

26. T.D. Cohen, J.L. Friar, G.A. Miller and U. van Kolck, Phys. Rev. C 53 (1996) 2661 arXiv:nucl-th/9512036.

27. C. da Rocha, G. Miller and U. van Kolck, Phys. Rev. C 61 (2000) 034613 arXiv:nucl-th/9904031.

28. C. Hanhart, U. van Kolck, and G.A. Miller, Phys. Rev. Lett. 85 (2000) 2905 arXiv:nucl-th/0004033.

29. C. Hanhart and N. Kaiser, Phys. Rev. C 66 (2002) 054005 arXiv:nucl-th/0208050.

30. C. Hanhart, Phys. Rept. 397, 155 (2004) arXiv:hep-ph/0311341.

31. D. S. Koltun and A. Reitan, Phys. Lett. 141 (1966) 1413.

32. J. A. Niskanen, Nucl. Phys. A 298 (1978) 417.

33. C. Hanhart, J. Haidenbauer, O. Krehl and J. Speth, Phys. Lett. B 444 (1998) 25 arXiv:nucl-th/9808020.

34. V. Lensky, V. Baru, J. Haidenbauer, C. Hanhart, A. E. Kudryavtsev and U.G. Meißner, Phys. Lett. B 648 (2007) 46 arXiv:nucl-th/0608042.

35. V. Baru, J. Haidenbauer, C. Hanhart, A. E. Kudryavtsev, V. Lensky and U.G. Meißner, Phys. Lett. B 659 (2008) 184 arXiv:0706.4023 [nucl-th]].

36. V. Baru, J. Haidenbauer, C. Hanhart, A. E. Kudryavtsev, V. Lensky and U.G. Meißner, arXiv:0711.2743 [nucl-th].

37. J. Gasser, V. E. Lyubovitskij and A. Rusetsky, Phys. Rept. 456, 167 (2008) arXiv:0711.3522 [hep-ph]].

38. E. Epelbaum, A. Nogga, W. Gloeckle, H. Kamada, U.-G. Meißner and H. Witala, Phys. Rev. C 66 (2002) 064001 arXiv:nucl-th/0208023.

39. A. Gardestig, Phys. Rev. C 74 (2006) 017001 arXiv:nucl-th/0604035.

40. T. S. Park et al., Phys. Rev. C 67 (2003) 055206 arXiv:nucl-th/0208055].

41. A. Gardestig and D. R. Phillips, Phys. Rev. Lett. 96 (2006) 232301 arXiv:nucl-th/0603045.

42. S. X. Nakamura, Phys. Rev. C 77 (2008) 054001 arXiv:0709.1239 [nucl-th]].

43. R.W. Flammang et al., Phys. Rev. C 58, 916 (1998).

44. H.O. Meyer et al., Phys. Rev. Lett. 83 (1999) 5439; H. O. Meyer et al., Phys. Rev. C 63, 064002 (2001).

45. P. N. Deepak, J. Haidenbauer and C. Hanhart, Phys. Rev. C 72, 024004 (2005) arXiv:hep-ph/0503228.

46. A. Kacharava et al., "Spin physics from COSY to FAIR," arXiv:nucl-ex/0511028

47. S. Weinberg, Trans. New York Acad. Sci. 38 (1977) 185.

48. U.-G. Meißner and S. Steininger, Phys. Lett. B 419 (1998) 403 arXiv:hep-ph/9709453.

49. A. K. Opper et al., Phys. Rev. Lett. 91 (2003) 212302 arXiv:nucl-ex/0306027.

50. E. J. Stephenson et al., Phys. Rev. Lett. 91 (2003) 142302 arXiv:nucl-ex/0305032.

51. U. van Kolck, J. A. Niskanen and G. A. Miller, Phys. Lett. B 493, 65 (2000) arXiv:nucl-th/0006042.

52. A. Nogga et al., Phys. Lett. B 639, 465 (2006) arXiv:nucl-th/0602003; A. Gardestig et al., Phys. Rev. C 69, 044606 (2004) arXiv:nucl-th/0402021]; T. A. Lahde and G. A. Miller, Phys. Rev. C 75, 055204 (2007) [Erratum-ibid. C 77, 019904 (2008)] arXiv:nucl-th/0703021; G. A. Miller, A. K. Opper and E. J. Stephenson, Ann. Rev. Nucl. Part. Sci. 56 (2006) 253 arXiv:nucl-ex/0602021.

53. F. K. Guo, C. Hanhart, S. Krewald and U.-G. Meißner, Phys. Lett. B 666, 251 (2008) arXiv:0806.3374 [hep-ph]].

54. H. H. Adam et al. [WASA-at-COSY Collaboration], "Proposal for the Wide 
Angle Shower Apparatus (WASA) at COSY-Juelich - 'WASA at COSY'," arXiv:nucl-ex/0411038

55. L. Alvarez-Ruso, E. Oset and E. Hernandez, Nucl. Phys. A 633 (1998) 519 arXiv:nucl-th/9706046.

56. W. Brodowski et al., Phys. Rev. Lett. 88 (2002) 192301; J. Johanson et al., Nucl. Phys. A 712 (2002) 75.

57. J. Patzold et al., Phys. Rev. C 67 (2003) 052202 arXiv:nucl-ex/0301019.

58. S. A. El-Bary et al. [COSY-TOF Collaboration], arXiv:0806.3870 [nucl-ex], Eur. Phys. J. A in print.

59. O. Krehl, C. Hanhart, S. Krewald and J. Speth, Phys. Rev. C 62 (2000) 025207 arXiv:nucl-th/9911080.

60. S. Schneider, S. Krewald and U.-G. Meißner, Eur. Phys. J. A 28 (2006) 107 arXiv:nucl-th/0603040.

61. Z. Ouyang, J. J. Xie, B. S. Zou and H. S. Xu, arXiv:0808.3257 [nucl-th].

62. M. Goldberger and K. Watson, Collision Theory, Wiley, New York, 1964.

63. A. Gasparyan, J. Haidenbauer and C. Hanhart, Phys. Rev. C 72 (2005) 034006 arXiv:nucl-th/0506067.

64. A. Gasparyan, J. Haidenbauer, C. Hanhart and J. Speth, Phys. Rev. C 69 (2004) 034006 arXiv:hep-ph/0311116.

65. C. Hanhart and K. Nakayama, Phys. Lett. B 454 (1999) 176 arXiv:nucl-th/9809059.

66. M. Pfeiffer et al., Phys. Rev. Lett. 92 (2004) 252001 arXiv:nucl-ex/0312011].

67. B. Mayer et al., Phys. Rev. C 53 (1996) 2068.

68. T. Mersmann et al., Phys. Rev. Lett. 98 (2007) 242301 arXiv:nucl-ex/0701072.

69. J. Smyrski et al., Phys. Lett. B 649 (2007) 258 arXiv:nucl-ex/0702043].

70. A. Sibirtsev, J. Haidenbauer, C. Hanhart and J. A. Niskanen, Eur. Phys. J. A 22 (2004) 495 arXiv:nucl-th/0310079; A. Sibirtsev, J. Haidenbauer, J. A. Niskanen and U.-G. Meißner, Phys. Rev. C 70 (2004) 047001 arXiv:nucl-th/0407073].

71. C. Wilkin et al., Phys. Lett. B 654 (2007) 92 arXiv:0707.1489 [nucl-ex]].

72. C. Hanhart, Phys. Rev. Lett. 94 (2005) 049101 arXiv:hep-ph/0408204.

73. G. Zweig, CERN Report 8419/Th, 412, 1964; S. Okubo, Phys. Lett. 5 (1963) 165; J. Iizuka, Prog. Theor. Phys. Suppl. 37 (1966) 21.

74. J. R. Ellis, M. Karliner, D. E. Kharzeev and M. G. Sapozhnikov, Phys. Lett. B 353 (1995) 319 arXiv:hep-ph/9412334.

75. A. I. Titov, B. Kämpfer and B. L. Reznik, Eur. Phys. J. A 7 (2000) 543 arXiv:nucl-th/0001027.

76. A. Sibirtsev, J. Haidenbauer and U. G. Meissner, Eur. Phys. J. A 27 (2006) 263 arXiv:nucl-th/0512055.

77. K. Nakayama, A. Szczurek, C. Hanhart, J. Haidenbauer and J. Speth, Phys. Rev. C 57 (1998) 1580 arXiv:nucl-th/9802013.

78. K. Nakayama, J. W. Durso, J. Haidenbauer, C. Hanhart and J. Speth, Phys. Rev. C 60 (1999) 055209 arXiv:nucl-th/9904040.

79. A. I. Titov, B. Kämpfer and B. L. Reznik, Phys. Rev. C 65 (2002) 065202 arXiv:nucl-th/0102032.

80. K. Tsushima and K. Nakayama, Phys. Rev. C $\quad 68$ (2003) 034612 arXiv:nucl-th/0304017.

81. M. Abdel-Bary et al., Phys. Lett. B 647 (2007) 351; S.Barsov et al., Eur. Phys. J. A31, 95 (2007); S.Barsov et al., Eur.Phys.J. A 21, 521 (2004); M.Hartmann et al., Phys. Rev. Lett. 96, 242301 (2006); Y.Maeda et al., Phys. Rev. Lett. 97, 142301 (2006); Y.Maeda et al., Phys. Rev. C 77, 015204 (2008). 